\title{
A homogenization approach to flashing ratchets
}

\author{
Benoit Perthame and Panagiotis E. Souganidis
}

\begin{abstract}
The paper continues the program of the authors to develop a mathematical framework to understand and characterize the notion of "asymmetric" potentials, which has been introduced to explain how molecular motors work, considering flashing ratchets, i.e., molecules diffusing in a potential with periodic switches. The mathematical model is a Fokker-Planck equation with a space-time periodic potential and diffusion of order of magnitude compatible with the period of the potential. After performing a homogenization analysis of the problem the "asymmetric" potentials are characterized by the property that the solution, which models the molecule density, concentrates on one end of the domain. Finally explicit examples are presented exhibiting that the concentration phenomena (motor effect) takes place are presented. The proof uses techniques from the theory of viscosity solutions for the HamiltonJacobi equation which, in the homogenization limit, defines the effective hamiltonian.
\end{abstract}

Mathematics Subject Classification (2000). 35B25, 35B27, 49L25, 92C05.

Keywords. Flashing ratchets, Molecular motors, Homogenization, Singular perturbations, Hamilton-Jacobi equations, Viscosity solutions, Floquet eigenfunction.

\section{Introduction}

We continue our program to develop a mathematical framework to understand and characterize the notion of "asymmetric" potentials that has been introduced (see $[5,11,19,26,28,35]$ ) by physicists to explain motor effects, i.e., how molecular motors work. In our previous work [33,34] we studied models with conventional kinesis, while in this note we consider the so-called flashing ratchets models. The term "flashing ratchets" refers to the case of molecules 
diffusing in a potential with space-time periodic switches and diffusion of size comparable to the period of the potential. Following the setting proposed in $[13,14,27,29]$ (see [20] for another), the molecules are presented by the density $n_{\varepsilon}(x, t)$ which evolves by the Fokker-Planck equation

$$
\left\{\begin{array}{l}
n_{\varepsilon, t}-\varepsilon n_{\varepsilon, x x}-\left(W_{y}\left(\frac{x}{\varepsilon}, \frac{t}{\varepsilon}\right) n_{\varepsilon}\right)_{x}=0 \text { in } x \in(0,1) \times \mathbb{R}, \\
\varepsilon n_{\varepsilon, x}+W_{y}\left(\frac{x}{\varepsilon}, \frac{t}{\varepsilon}\right) n_{\varepsilon}=0 \text { at } x=0,1 \text { and } t \in \mathbb{R}, \\
n_{\varepsilon} \text { is } \varepsilon \text {-periodic in } t, n_{\varepsilon}>0, \text { and } \int_{0}^{1} n_{\varepsilon}(x, t) d x=1 \text { for all } t \in \mathbb{R},
\end{array}\right.
$$

which is a Floquet eigenproblem that has a unique positive solution (up to multiplication by a constant which explains the unit mass normalization).

It follows from the relative entropy method that, for all initial data, the solution of the associated initial value problem approaches, as $t \rightarrow \infty$, a multiple of the time-periodic solution of (1.1). This is the reason why we restrict our study to the time-periodic solutions. The zero flux boundary conditions imply that the total number of molecules is constant for all time, a fact that allows us to normalize the total mass. Finally $W(y, \tau)$ is the potential in which the molecules are moving.

We assume that

$$
W \text { is } C^{2}(\mathbb{R} \times \mathbb{R}) \text { and } 1-\text { periodic in } y \text { and } \tau,
$$

and we choose

$$
\varepsilon=1 / N \text { for some positive integer } N \text {, }
$$

so that

$$
W\left(\frac{1}{\varepsilon}, \tau\right)=W(0, \tau) \text { and } W_{y}\left(\frac{1}{\varepsilon}, \tau\right)=W_{y}(0, \tau)
$$

A particular example of such potential is $W(y, \tau)=V(y) \psi(\tau)$ where $\psi$ and $V$ are smooth and 1-periodic and $\psi$ acts as a switch, i.e., for some $\tau_{0} \in(0,1)$

$$
\psi(\tau)=\left\{\begin{array}{l}
0 \quad \text { for } 0 \leq \tau \leq \tau_{0} \\
>0 \text { for } \tau_{0}<\tau \leq 1
\end{array}\right.
$$

Several molecular motor models were analyzed, for fixed $\varepsilon$, in $[13,14,20$, $27,29]$ using arguments from optimal transportation or ordinary differential equations. The typical results about biomotors obtained in $[13,27,33]$, without periodic potentials, are that, for small diffusion $\varepsilon$ and under some precise asymmetry assumptions on the potential and rates, the solutions tend to concentrate, as $\varepsilon \rightarrow 0$, as Dirac masses at either $x=0$ or $x=1$. This behavior is referred to as "motor effect".

In [34], we proposed to set the problem, in the context of conventional kinesis, in the homogenization framework, and showed that the motor effect holds for much more general potentials.

Along this line, the question we are asking here is for which space-time periodic potentials $W$ does (1.1) exhibit motor effect as $\varepsilon \rightarrow 0$. This is a 
homogenization problem that we treat using the WKB method. More precisely, we are investigating for which $W$, satisfying (1.2), we have,

$$
n_{\varepsilon}=\exp \left[-\frac{1}{\varepsilon}(R+o(1))\right],
$$

with an effective rate $R$ having a strict minimum at one end of the domain $(0,1)$. This gives the orientation of the molecular transport. Indeed, if $\delta_{a}$ denotes the Dirac mass at $x=a$, the above statement together with the unit mass normalization imply that

$$
n_{\varepsilon} \underset{\varepsilon \rightarrow 0}{\longrightarrow} \delta_{0} \quad \text { or } \quad \delta_{1}
$$

Our approach is based on the analysis of the homogenization limit, as $\varepsilon \rightarrow 0$, of the problem satisfied by the rate functions $R_{\varepsilon}$ defined by the classical transformation

$$
n_{\varepsilon}=\exp \left(-\frac{1}{\varepsilon} R_{\varepsilon}\right) .
$$

The limit $R$ of $R_{\varepsilon}$ 's solves, in the Crandall-Lions viscosity sense, an averaged Hamilton-Jacobi equation. We show that the critical condition for motor effect is that the homogenized Hamiltonian has a non-zero root.

Viscosity solutions are the correct class of weak solutions for first- and second-order fully nonlinear degenerate elliptic pde. We refer to [18] and the references therein for a good introduction to the theory. Our arguments combine ideas from the methods used to study front propagation and large deviations [6,23], Dirac concentrations [8] and homogenization [22,30].

There are other works that looked at space-time homogenization for initial value problems leading to concentration in finite time. For example, [21] considered traveling speeds for coefficients oscillating in $t / \varepsilon^{2}$ scale, while [2, $24]$ looked at the homogenization of parabolic equations in bounded domains when, among other cases, the coefficients oscillate as $t / \varepsilon$, and $[3,15,16]$ studied time-oscillatory perforated domains.

The main result is:

Theorem 1.1. We assume (1.2), (1.3) and let $n_{\varepsilon}$ satisfy (1.1). Then:

(i) Up to subsequences, the rates $R_{\varepsilon}$ defined in (1.6) converge, locally uniformly in $(0,1) \times \mathbb{R}$ to a time independent $R \in C((0,1))$.

(ii) There exists a convex effective Hamiltonian $\bar{H}(p)$, defined in Sect. 3, such that, in the viscosity sense,

$$
\bar{H}\left(R_{x}\right)=0 \quad \text { in }(0,1) .
$$

(iii) If the potential $W$ is asymmetric (in the sense of Definition 3.1 below), then $R_{x}=\bar{p}$, the only non-zero constant solution of $\bar{H}(p)=0$. Consequently, as $\varepsilon \rightarrow 0, n_{\varepsilon} \rightarrow \delta_{0}$ if $\bar{p}>0$ and $n_{\varepsilon} \rightarrow \delta_{1}$ if $\bar{p}<0$.

The last statement characterizes the asymmetric potentials, i.e., potentials for which the motor effect holds, because the molecules concentrate on one end of the interval $(0,1)$. It does not provide, however, either an explicit form for such potentials or a property that is easy to check. On the other hand 
it is possible to show, using perturbation arguments, that "asymmetry" is a generic property. We present such a construction later is the paper. Similar statements can be found in [17].

Another approach to model molecular motors is to consider the problem on the whole line and to seek traveling wave solutions or, more precisely, pulsating waves in the terminology of [9]. The issue is then to compute the asymptotic propagation speed for long times and to investigate the occurrence or not of concentration effects. This has been studied, for example, in $[10,17]$ for the case of flashing ratchets.

Finally we refer to $[1,4,12]$ for related homogenization problems concerning nonconservative, eigenvalue problems where the eigenvalue itself is also homogenized. This is related to what happens when (1.3) does not hold.

The novelties of Theorem 1.1 are the estimates leading to first conclusion, the identification of $\bar{H}$ and the selection of the unique $R_{x}$. The latter is nontrivial and it is accomplished using a variation of an argument, introduced by the authors in [34], which uses the adjoint problem to "record" some information at the $\varepsilon$-level which is crucial in identifying the unique limit.

The paper is organized as follows. The uniform convergence is proved in Sect. 2, the homogenized equation is derived in Sect. 3, the concentration phenomenon is established in Sect. 4. Examples of potentials satisfying the asymmetry condition are given in Sect. 5 .

\section{The uniform convergence}

The rate function $R_{\varepsilon}$ satisfies

$$
\left\{\begin{array}{l}
R_{\varepsilon, t}-\varepsilon R_{\varepsilon, x x}+\left|R_{\varepsilon, x}\right|^{2}-W_{y} R_{\varepsilon, x}+W_{y y}=0 \text { in }(0,1) \times \mathbb{R} \\
R_{\varepsilon} \text { is } \varepsilon \text {-periodic in } t \text { and } \max _{\frac{1}{2} \leq x \leq \frac{3}{4}, t \in \mathbb{R}} R_{\varepsilon}(x, t)=1
\end{array}\right.
$$

This new normalization of $R_{\varepsilon}$, which is introduced only to simplify the argument, means that the unit mass property of $n_{\varepsilon}$ is lost. This, however, is not essential for the full argument. We prove next that the $R_{\varepsilon}$ 's converge, locally uniformly in $(0,1)$, to a time independent $R$. Actually, for reasons we indicate later on, we believe that the limit is uniform on the full interval $(0,1)$. Since this is not necessary for the proof we do not pursue it here.

The main difficulty with proving the local uniform convergence of the $R_{\varepsilon}$ 's is the lack of any estimates other than $L^{\infty}$. Indeed any obvious attempt to prove, for example, Lipschitz bounds, fails, since differentiating the equation gives rise to $1 / \varepsilon$ dependent terms.

The usual way in the theory of viscosity solutions (see $[7,25]$ ) to avoid such difficulties is to use the so-called "half-relaxed" limits

$$
R^{*}(x, t)=\limsup _{y \rightarrow x, s \rightarrow t} R_{\varepsilon}(y, s), \quad R_{*}(x, t)=\liminf _{y \rightarrow x, s \rightarrow t} R_{\varepsilon}(y, s) .
$$

Once the equation satisfied by $R^{*}$ and $R_{*}$ is identified, one relies typically on the comparison principle of this equation to show that actually $R^{*}=R_{*}$ and, hence, that the limit of the $R_{\varepsilon}$ 's exists. 
Although in the problem at hand is not difficult to identify the limiting equation (we do this in the next section) in $(0,1)$, the issue is that we do not know much about the boundary conditions at the $\varepsilon=0$ level, something that is essential to have any comparison result.

Following an idea introduced in [32] for time-independent problems, it is possible, using the Harnack inequality, to go around this serious difficulty and prove that $R^{*}=R_{*}$ without any comparison result for the limiting problem.

An easy variant of the parabolic Harnack inequality [31] states that, for all $\varepsilon>0$ and $T>0$, there exists a constant $C>0$, which depends on the $L^{\infty}$-norm of the coefficients but not $\varepsilon$, such that, for all $x, y, s, t$ such that

$$
\varepsilon \leq s<t \leq T \text { and } \varepsilon<x, y<1-\varepsilon,
$$

we have

$$
\log \left(\frac{n_{\varepsilon}(y, s)}{n_{\varepsilon}(x, t)}\right) \leq \frac{C}{\varepsilon}\left[\frac{|x-y|^{2}}{t-s}+(t-s)+\varepsilon\right] .
$$

This inequality is itself the rescaled version of the standard Harnack inequality (see [31]) in the box

$$
1 \leq s<t \leq T / \varepsilon \text { and } 1<x, y<1 / \varepsilon-1
$$

for $u(y, \tau)=n_{\varepsilon}(\varepsilon y, \varepsilon \tau)$ that satisfies the, independent of $\varepsilon$, Fokker-Planck equation

$$
u_{\tau}-u_{y y}-\left(W_{y} u\right)_{y}=0 \text { in }(0,1 / \varepsilon) \times \mathbb{R} .
$$

It is now immediate from (2.3) that the rate functions $R_{\varepsilon}$ 's satisfy, for all $x, y, s, t$ as in (2.2),

$$
R_{\varepsilon}(x, t) \leq R_{\varepsilon}(y, s)+C\left[\frac{|x-y|^{2}}{t-s}+(t-s)+\varepsilon\right] .
$$

This is the basis of our proof. Notice that because of the zero flux boundary condition, we expect this inequality to be true up to the boundary, as in [32] for the elliptic case, a fact which would yield the uniform convergence up to the boundary.

We now argue with the previously defined "half-relaxed" limits, which exist in $(0,1) \times(0, T)$ because of the normalization of the $R_{\varepsilon}$ 's and the Harnack inequality. Indeed taking the limsup on the left hand side and the liminf on the right hand side of (2.4), we find that, for all $x, s, t$ as in (2.2),

$$
R^{*}(x, t) \leq R_{*}(x, s)+C(t-s) .
$$

Because the $R_{\varepsilon}$ 's are $\varepsilon$-time periodic, we immediately conclude that $R^{*}$ and $R_{*}$ are time independent, i.e., for all $x \in(0,1)$,

$$
R^{*}(x, t)=R^{*}(x) \quad \text { and } \quad R_{*}(x, t)=R_{*}(x) .
$$

It then follows that, for all $x \in(0,1)$ and $s \leq t$,

$$
R^{*}(x) \leq R_{*}(x)+C(t-s) .
$$


Finally letting $s \rightarrow t$ we obtain $R^{*} \leq R_{*}$ in $(0,1)$, which yields, by the very construction of these functions, that $R^{*}=R_{*}$ and thus the uniform convergence of $R_{\varepsilon}$ 's to $R$ on each subinterval of $(0,1)$. This concludes the proof of the first part of Theorem 1.1.

\section{The homogenized equation}

\subsection{The cell problem and flux properties}

The cell problem for (1.1) consists in finding, for each $p \in \mathbb{R}$, the effective Hamiltonian $\bar{H}(p)$ which is the first eigenvalue for the equation

$$
\left\{\begin{array}{l}
\chi_{\tau}-\chi_{y y}-\left(W_{y}(y, \tau) \chi\right)_{y}=-\bar{H}(p) \chi \text { in }(0,1) \times \mathbb{R} \\
\chi>0, \quad \chi e^{p y} \text { is 1-periodic in }(y, \tau) \text { and } \int_{0}^{1} \int_{0}^{1} \chi d \tau d y=1,
\end{array}\right.
$$

which is again a Floquet problem that has (see [21]) a unique solution up to multiplication by a constant.

It follows that (see $[30,34]$ for the elliptic and parabolic cases respectively)

$$
\bar{H}(0)=0 \text { and } \bar{H}(\cdot) \text { is convex. }
$$

Following the elliptic setting of [34] we proceed with the notion of asymmetric potentials. Observe that, in view of the convexity statement (3.2), in addition to $0, \bar{H}$ can have at most one more root and this is equivalent to $\bar{H}^{\prime}(0) \neq 0$.

We have:

Definition 3.1. A periodic potential $W(\cdot, \cdot)$ is asymmetric if there is a non-zero root $\bar{p}$ of $\bar{H}(\cdot)=0$.

As in [34] we look at the total flux in (3.1) in order to select the correct $p$. Note that, since the flux vanishes for $n_{\varepsilon}$, we can expect something similar for $\chi$.

The total flux is defined by

$$
\mathcal{F}(p)=\int_{0}^{1} F_{p}(\tau) d \tau
$$

where

$$
F_{p}(\tau)=\chi_{y}(0, \tau)+W_{y}(0, \tau) \chi(0, \tau) .
$$

Integrating (3.1) with respect to $y$ we find

$$
\begin{aligned}
\frac{d}{d \tau} \int_{0}^{1} \chi(y, \tau) d y & =\chi_{y}(1, \tau)+W_{y}(0, \tau) \chi(1, \tau)-F_{p}(\tau)+\bar{H}(p) \int_{0}^{1} \chi(y, \tau) d y \\
& =F_{p}(\tau)\left(e^{-p}-1\right)+\bar{H}(p) \int_{0}^{1} \chi(y, \tau) d y .
\end{aligned}
$$

The periodicity in time and the normalization in (3.1) yield

$$
\left(1-e^{-p}\right) \mathcal{F}(p)=\bar{H}(p) .
$$


This identity at $p=0$ says nothing but $\bar{H}(0)=0$ which we already know. Because $1-e^{-p} \neq 0$ for $p \neq 0,(3.5)$ also has a useful consequence for our analysis.

The following result is proved as in [34], hence we omit its proof here.

Lemma 3.2. The potential $W$ is asymmetric if and only if the following three equivalent conditions are satisfied

(i) $\mathcal{F}(\bar{p})=0$,

(ii) $\mathcal{F}(0) \neq 0$,

(iii) $\frac{d}{d p} \bar{H}(0) \neq 0$.

It is also useful for arguments to follow as well as to put the problem in the context of homogenization to observe that, if $\phi$ is defined by $\chi(y)=e^{-\phi(y)-p y}$, then

$$
\left\{\begin{array}{l}
\phi_{\tau}-\phi_{y y}+\left|\phi_{y}+p\right|^{2}+W_{y}(y, \tau) \phi_{y}-W_{y y}(y, \tau)=\bar{H}(p) \text { in }(0,1) \times \mathbb{R}, \\
\phi \text { is } 1-\text { periodic of period1 in }(y, \tau) .
\end{array}\right.
$$

\subsection{The limiting Hamilton-Jacobi equation}

The effective Hamiltonian determines the limit of the $R_{\varepsilon}$ 's in the sense of Theorem 1.1. Indeed using the by now standard perturbed test function method (see [22]), we find that all possible limits $R$ of the $R_{\varepsilon}$ 's are solutions of (1.7).

We first present the method in full generality ignoring that $R$ is time independent. We depart from (2.1) and prove the subsolution property (the supersolution characterization follows similarly).

To this end let $\Phi \in C^{2}(\mathbb{R} \times \mathbb{R})$ be a test function and assume that $\left(x_{0}, t_{0}\right)$ and $\left(x_{\varepsilon}, t_{\varepsilon}\right)$ are such that

$$
\max _{x, t}[R-\Phi](x, t)=[R-\Phi]\left(x_{0}, t_{0}\right)
$$

and

$$
\max _{x, t}\left[R_{\varepsilon}-\Phi-\varepsilon \phi\left(\frac{\dot{-}}{\varepsilon}, \frac{\dot{\varepsilon}}{\varepsilon}\right)\right](x, t)=\left[R_{\varepsilon}-\Phi-\varepsilon \phi\left(\frac{\dot{-}}{\varepsilon} \frac{\dot{\varepsilon}}{\varepsilon}\right)\right]\left(x_{\varepsilon}, t_{\varepsilon}\right),
$$

where the $p$ which defines $\phi$ is chosen as $p=\Phi_{x}\left(x_{0}, t_{0}\right)$. As usual, we may assume, extracting if necessary a subsequence, that, as $\varepsilon \rightarrow 0,\left(x_{\varepsilon}, t_{\varepsilon}\right) \rightarrow$ $\left(x_{0}, t_{0}\right)$.

Evaluating (2.1) at the point $\left(x_{\varepsilon}, t_{\varepsilon}\right)$ and using that, in view of (3.8), at $\left(x_{\varepsilon}, t_{\varepsilon}\right)$,

$$
\begin{aligned}
R_{\varepsilon, t} & =\Phi_{t}+\phi_{\tau}\left(\frac{\dot{-}}{\varepsilon}, \frac{\dot{ }}{\varepsilon}\right), \quad R_{\varepsilon, x}=\Phi_{x}+\phi_{y}\left(\frac{\dot{-}}{\varepsilon}, \frac{\dot{-}}{\varepsilon}\right) \quad \text { and } \\
R_{\varepsilon, x x} & \leq \Phi_{x x}+\frac{1}{\varepsilon} \phi_{y y}\left(\frac{\cdot}{\varepsilon}, \frac{\cdot}{\varepsilon}\right)
\end{aligned}
$$


we arrive at the inequality

$$
\begin{aligned}
& \Phi_{t}\left(x_{\varepsilon}, t_{\varepsilon}\right)+\phi_{\tau}\left(\frac{x_{\varepsilon}}{\varepsilon}, \frac{t_{\varepsilon}}{\varepsilon}\right)-\varepsilon \Phi_{x x}\left(x_{\varepsilon}, t_{\varepsilon}\right)-\phi_{y y}\left(\frac{x_{\varepsilon}}{\varepsilon}, \frac{t_{\varepsilon}}{\varepsilon}\right) \\
& +\left|\Phi_{x}\left(x_{\varepsilon}, t_{\varepsilon}\right)+\phi_{y}\left(\frac{x_{\varepsilon}}{\varepsilon}, \frac{t_{\varepsilon}}{\varepsilon}\right)\right|^{2}-W_{y}\left(\frac{x_{\varepsilon}}{\varepsilon}, \frac{t_{\varepsilon}}{\varepsilon}\right)\left[\Phi_{x}\left(x_{\varepsilon}, t_{\varepsilon}\right)+\phi_{y}\left(\frac{x_{\varepsilon}}{\varepsilon}, \frac{t_{\varepsilon}}{\varepsilon}\right)\right] \\
& -W_{y y}\left(\frac{x_{\varepsilon}}{\varepsilon}, \frac{t_{\varepsilon}}{\varepsilon}\right) \leq 0,
\end{aligned}
$$

and, hence, for $\varepsilon$ sufficiently small

$$
\begin{gathered}
\Phi_{t}\left(x_{0}, t_{0}\right)+\phi_{\tau}\left(\frac{x_{\varepsilon}}{\varepsilon}, \frac{t_{\varepsilon}}{\varepsilon}\right)-\phi_{y y}\left(\frac{x_{\varepsilon}}{\varepsilon}, \frac{t_{\varepsilon}}{\varepsilon}\right)+\left|\Phi_{x}\left(x_{0}, t_{0}\right)+\phi_{y}\left(\frac{x_{\varepsilon}}{\varepsilon}, \frac{t_{\varepsilon}}{\varepsilon}\right)\right|^{2} \\
-W_{y}\left(\frac{x_{\varepsilon}}{\varepsilon}, \frac{t_{\varepsilon}}{\varepsilon}\right)\left[\Phi_{x}\left(x_{0}, t_{0}\right)+\phi_{y}\left(\frac{x_{\varepsilon}}{\varepsilon}, \frac{t_{\varepsilon}}{\varepsilon}\right)\right]-W_{y y}\left(\frac{x_{\varepsilon}}{\varepsilon}, \frac{t_{\varepsilon}}{\varepsilon}\right) \leq o(1),
\end{gathered}
$$

which, using (3.6), leads to

$$
\Phi_{t}\left(x_{0}, t_{0}\right)+\bar{H}\left(\Phi_{x}\left(x_{0}, t_{0}\right)\right) \leq o(1) .
$$

Letting $\varepsilon \rightarrow 0$, we find that $R$ is a subsolution of the evolution HamiltonJacobi equation for the effective hamiltonian.

For the case at hand we know that $R$ is time independent. Therefore we just choose test functions $\Phi$ that depend only on $x$. We find that, as $\varepsilon \rightarrow$ $0, x_{\varepsilon} \rightarrow x_{0}$ while the limit information on $t_{\varepsilon}$ becomes irrelevant as it is clear from the above derivation of (1.7).

\section{The concentration phenomena}

The main difficulty in proving the concentration phenomenon (the third part of Theorem 1.1) is that there are several viscosity solutions to (1.7) including constants. As already mentioned, this is because we do not know how to derive the boundary conditions in the limit. It is therefore necessary to find a different way to identify the "correct" limit of the $R_{\varepsilon}$ 's. Following an idea already introduced in [34], we use the zero flux property.

To this end, let $\bar{p}$ be the unique non-zero root of $\bar{H}=0$ and consider the dual Floquet equation to (3.1), which reads

$$
\left\{\begin{array}{l}
-\zeta_{\tau}-\zeta_{y y}+W_{y} \zeta_{y}=0 \text { in } \mathbb{R} \times \mathbb{R} \\
\zeta>0, \text { and }(y, \tau) \rightarrow \zeta(y, \tau) e^{-\bar{p} y} \text { is } 1-\text { periodic in }(y, \tau) .
\end{array}\right.
$$

Using $\zeta$ we construct a solution $\zeta_{\varepsilon}$ to the dual parabolic equation to (1.1) (but with different boundary conditions)

$$
\left\{\begin{array}{l}
-\frac{\partial}{\partial t} \zeta_{\varepsilon}-\varepsilon \zeta_{\varepsilon, x x}+W_{y}\left(\frac{x}{\varepsilon}, \frac{t}{\varepsilon}\right) \zeta_{\varepsilon, x}=0 \text { in }(0,1) \times \mathbb{R}, \\
\zeta_{\varepsilon}>0, \zeta_{\varepsilon}(1, t)=e^{\bar{p} / \varepsilon} \zeta_{\varepsilon}(0, t), \zeta_{\varepsilon, x}(1, t)=e^{\bar{p} / \varepsilon} \zeta_{\varepsilon, x}(0, t) .
\end{array}\right.
$$

Indeed, recalling the $N \varepsilon=1$, we define

$$
\left.\zeta_{\varepsilon}(x, t)=\zeta_{\left(\frac{x}{\varepsilon}\right.}, \frac{t}{\varepsilon}\right) e^{\bar{p} n} \quad \text { for } \varepsilon n \leq x<\varepsilon(n+1) \text { and } 0 \leq n \leq N-1 \text {, }
$$


which is, in view of the boundary condition on $\zeta$, a $C^{2}$ solution to (4.2) in $(\varepsilon n, \varepsilon(n+1)) \times \mathbb{R}$ for each $n$ as above.

Integrating by parts we get

$$
\frac{d}{d t} \int_{0}^{1}\left(\zeta_{\varepsilon} n_{\varepsilon}\right)(x, t) d x=\varepsilon \zeta_{\varepsilon} n_{\varepsilon, x}-\varepsilon n_{\varepsilon} \zeta_{\varepsilon, x}+\left.\zeta_{\varepsilon} n_{\varepsilon} W_{y}\right|_{0} ^{1},
$$

and, in view of the zero flux boundary condition, we conclude that

$$
\frac{d}{d t} \int_{0}^{1}\left(\zeta_{\varepsilon} n_{\varepsilon}\right)(x, t) d x=-\left.\varepsilon n_{\varepsilon} \zeta_{\varepsilon, x}\right|_{0} ^{1} .
$$

Finally integrating in time and using the time periodicity we find

$$
\int_{0}^{1} n_{\varepsilon}(1, t) \zeta_{\varepsilon, x}(1, t) d t=\int_{0}^{1} n_{\varepsilon}(0, t) \zeta_{\varepsilon, x}(0, t) d t
$$

and, thus,

$$
e^{\bar{p} / \varepsilon} \int_{0}^{1} n_{\varepsilon}(1, t) \zeta_{y}\left(0, \frac{t}{\varepsilon}\right) d t=\int_{0}^{1} n_{\varepsilon}(0, t) \zeta_{y}\left(0, \frac{t}{\varepsilon}\right) d t .
$$

At this point we need the following technical lemma which we prove at the end of this Section.

Lemma 4.1. For each $\tau \in \mathbb{R}, y \mapsto \zeta(y, \tau)$ is strictly increasing if $\bar{p}>0$ and strictly decreasing if $\bar{p}<0$. Therefore, there exist $\beta_{+}>\beta_{-}>0$ such that, for all $\tau \in \mathbb{R}$,

$$
\beta_{-} \leq\left|\zeta_{y}(0, \tau)\right| \leq \beta_{+}
$$

With the lemma at hand we can conclude. Indeed assume, for definiteness, that $\bar{p}>0$. Using the ansatz (1.4) and $R=R(x)$, we deduce

$$
\beta_{-} e^{\frac{-\bar{p}+R(1)+o(1)}{\varepsilon}} \geq \beta_{+} e^{\frac{R(0)+o(1)}{\varepsilon}},
$$

and, hence,

$$
R(1) \geq R(0)+\bar{p}
$$

Since all viscosity solutions to (1.7) have derivatives 0 or $\bar{p}$, in view of the above, we must have

$$
R(x)=R(0)+\bar{p} x .
$$

The proof is now complete.

Proof of Lemma 4.1. We only consider $\bar{p}>0$ since the other case follows similarly.

Set $z=\zeta_{y}$. Differentiating (4.1) with respect to $y$, we find that

$$
\left\{\begin{array}{l}
-\frac{\partial}{\partial \tau} z-z_{y y}+\left(W_{y} z\right)_{y}=0 \text { in } \mathbb{R} \times \mathbb{R} \\
(y, \tau) \rightarrow z(y, \tau) e^{-\bar{p} y} \text { is 1-periodic in }(y, \tau) .
\end{array}\right.
$$

The fact that the dual of the above problem is the cell problem (3.1) yields, in view of the Krein-Rutman theorem, that 0 is the principal eigenvalue and $z$ the associated eigenfunction. Therefore it has a sign. Since $\zeta(1, \tau)>$ 
$\zeta(1, \tau) e^{-\bar{p}}=\zeta(0, \tau)$, then $z=\zeta_{y}$ can only be positive and the statement is proved.

\section{Examples of asymmetric potentials/switches}

In [17] it is shown, for the traveling speed models of flashing ratchet, that there is an open set of data for which motor effect occurs. This is also the spirit of the construction of the potential/transition coefficients in [34] for standard kinesis where a generic perturbation of a symmetric potential is proved to yield motor effect.

Here we follow the latter approach, i.e., we perturb a class of "symmetric potentials" in the sense of Definition 3.1 to obtain the non-zero flux condition and, hence, "asymmetric" ones.

We begin with a straightforward calculation which shows how to find a potential $W$ being given a priori the solution $\chi$ to the cell problem (3.1) with $p=0$. Then, we introduce a class of symmetric potentials, i.e., potentials for which the corresponding total flux vanishes according to the Lemma 3.2. Finally we show that a perturbation yields non-zero flux and thus asymmetric potentials.

To this end, given an 1-space-time periodic function $\chi>0$ we find a periodic potential $W$ such that $\chi$ is a solution of the cell problem (3.1) with $p=0$. Indeed rewriting $(3.1)$ as

$$
\frac{\partial}{\partial \tau} \int_{0}^{y} \chi(z, \tau) d z-\chi_{y}(y, \tau)-W_{y}(y, \tau) \chi(y, \tau)=F_{0}(\tau),
$$

we compute the flux $F_{0}$ and $W_{y}$ and find

$$
F_{0}(\tau)=-\chi_{y}(0, \tau)-W_{y}(0, \tau) \chi(0, \tau)
$$

is the total flux already used in (3.4).

For $(y, \tau) \in(0,1) \times \mathbb{R}$, we define $W$ from $\chi$ and $F_{0}$ by

$$
W_{y}(y, \tau)=\frac{1}{\chi(y, \tau)} \frac{\partial}{\partial \tau} \int_{0}^{y} \chi(z, \tau) d z-(\ln \chi(y, \tau))_{y}-\frac{F_{0}(\tau)}{\chi(y, \tau)} .
$$

Since we are seeking 1 -space-time periodic potentials $W$ in $(y, \tau)$, for all $\tau \in \mathbb{R}$, we must have

$$
\int_{0}^{1} W_{y}(y, \tau) d y=0
$$

Hence the total flux is given by the formula

$$
F_{0}(\tau)=\left[\int_{0}^{1} \frac{1}{\chi(y, \tau)} d y\right]^{-1} \int_{0}^{1}\left[\frac{1}{\chi(y, \tau)} \frac{\partial}{\partial \tau} \int_{0}^{y} \chi(z, \tau) d z\right] d y
$$

We continue with a particular class of potentials (or solutions) with zero flux. 
Lemma 5.1. Potentials $W$ corresponding to $\chi$ 's of the form $\chi(y, \tau)=a(\tau) b(y)$ with $a>0, b>0$ and 1-periodic in $\tau$ and $y$ respectively are "symmetric", i.e., their total flux $\int_{0}^{1} F_{0}(\tau) d \tau$ vanishes. The total flux, however, does not vanish, and, hence, there is a motor effect, if $\chi(y, \tau)=a(\tau) b(y)+c(\tau)$ for appropriate choices of the 1-periodic in $\tau$ function $c(\cdot)$.

Proof. To prove that $\chi$ 's of the form $\chi=a b$ as above yield 0 total flux, we set $B(y)=\int_{0}^{y} b(z) d z$ and rewrite (5.3) as

$$
F_{0}(\tau)=\dot{a}(\tau) \mu \quad \text { for } \mu=\left[\int_{0}^{1} \frac{1}{b(y)} d y\right]^{-1} \int_{0}^{1} \frac{B(y)}{b(y)} d y
$$

In view of the periodicity, we conclude that

$$
\int_{0}^{1} F_{0}(\tau) d \tau=\mu(a(1)-a(0))=0 .
$$

Next we consider $\chi^{\prime}$ 's of the form

$$
\chi(y, \tau)=a(\tau) b(y)+c(\tau) \text { with } c \text { small and 1-periodic, }
$$

and show that a perturbation argument from the separated variable case makes the total flux nonzero.

Elementary calculations give

$$
\begin{aligned}
\int_{0}^{1} \frac{1}{\chi(y, \tau)} d y & =\frac{1}{a(\tau)} \int_{0}^{1} \frac{1}{b}-\frac{c(\tau)}{a^{2}(\tau)} \int_{0}^{1} \frac{1}{b^{2}}+O\left(c^{2}\right), \\
{\left[\int_{0}^{1} \frac{1}{\chi(y, \tau)} d y\right]^{-1} } & =\frac{a(\tau)}{\int_{0}^{1} \frac{1}{b}}+c(\tau) \frac{\int_{0}^{1} \frac{1}{b^{2}}}{\left(\int_{0}^{1} \frac{1}{b}\right)^{2}}+O\left(c^{2}\right),
\end{aligned}
$$

and

$$
\int_{0}^{1}\left[\frac{1}{\chi(y, \tau)} \frac{\partial}{\partial \tau} \int_{0}^{y} \chi(z, \tau) d z\right] d y=\frac{\dot{a}}{a} \int_{0}^{1} \frac{B}{b}-\frac{\dot{a} c}{a^{2}} \int_{0}^{1} \frac{B}{b^{2}}+\frac{\dot{c}}{a} \int_{0}^{1} \frac{y}{b}+O\left(c^{2}\right)
$$

where to keep the formulae a bit simpler we omitted, whenever possible, the integration variables.

Inserting the above in (5.3) we obtain

$$
F_{0}(\tau)=\dot{a} \mu+\frac{\dot{a} c}{a} \frac{\int_{0}^{1} \frac{1}{b^{2}}}{\left(\int_{0}^{1} \frac{1}{b}\right)^{2}} \int_{0}^{1} \frac{B}{b}-\frac{\dot{a} c}{a} \frac{\int_{0}^{1} \frac{B}{b^{2}}}{\int_{0}^{1} \frac{1}{b}}+\dot{c} \frac{\int_{0}^{1} \frac{y}{b}}{\int_{0}^{1} \frac{1}{b}}+O\left(c^{2}\right),
$$

and, after integrating in $\tau$,

$$
\int_{0}^{1} F_{0}(\tau) d \tau=\int_{0}^{1} \frac{\dot{a} c}{a} d \tau\left[\frac{\int_{0}^{1} \frac{1}{b^{2}}}{\left(\int_{0}^{1} \frac{1}{b}\right)^{2}} \int_{0}^{1} \frac{B}{b}-\frac{\int_{0}^{1} \frac{B}{b^{2}}}{\int_{0}^{1} \frac{1}{b}}\right]+O\left(c^{2}\right) .
$$

This flux does not vanish in general. To give an explicit example we choose, for small $\delta, c=\delta \dot{a}$ and $b$ such that

$$
\int_{0}^{1} \frac{1}{b^{2}} \int_{0}^{1} \frac{B}{b} \neq \int_{0}^{1} \frac{B}{b^{2}} \int_{0}^{1} \frac{1}{b}
$$


To check that such positive periodic functions $b$ exist, we may again choose a small perturbation of a constant, i.e., $b(y)=1+q(y)$ with $q$ small and $\int_{0}^{1} q(y) d y=0$. Set $Q(y)=\int_{0}^{y} q(z) d z$. Up to terms of order $O\left(q^{2}\right)$ we have to check that

$$
\int_{0}^{1}(y+Q(y))(1-q(y)) d y \neq \int_{0}^{1}(y+Q(y))(1-2 q(y)) d y
$$

which, in view of the facts that $Q^{\prime}=q$ and, thus $\left.\int(q Q)(y) d y=0\right)$ boils down to

$$
\int_{0}^{1} y q(y) d y \neq 0
$$

a property that allows for a large choice of $q$ and proves the Lemma.

\section{References}

[1] Allaire, G., Capdeboscq, Y.: Homogenization of a spectral problem in neutronic multigroup diffusion. Comput. Methods Appl. Mech. Eng. 187(1-2), 91-117 (2000)

[2] Allaire, G., Orive, R.: Homogenization of periodic non self-adjoint problems with large drift and potential. COCV 13, 735-749 (2007)

[3] Allaire, G., Piatnitsky, A.: Homogenization of nonlinear reaction-diffusion equation with a large reaction term. Annali dell'Universita di Ferrara 56, 141-161 (2010).

[4] Allaire, G., Raphael, A.-L.: Homogenization of a convection-diffusion model with reaction in a porous medium. C. R. Acad. Sci. Paris Ser. I 334, 523-528 (2007)

[5] Astumian, R.D., Hänggi, P.: Brownian motors. Phys. Today 33-39 (2002)

[6] Barles, G., Evans, L.C., Souganidis, P.E.: Wavefront propagation for reaction diffusion systems of PDE. Duke Math. J. 61, 835-858 (1990)

[7] Barles, G., Perthame, B.: Discontinuous solutions of deterministic optimal stopping time problems. RAIRO Model. Math. Anal. Num. 21(4), 557-579 (1987)

[8] Barles, G., Perthame, B.: Concentrations and constrained Hamilton-Jacobi equations arising in adaptive dynamics. In: Danielli, D. (ed.) Recent Developments in Nonlinear Partial Differential Equations. Contemp. Math., vol 439, pp. 57-68 (2007)

[9] Berestycki, H., Hamel, F.: Front propagation in periodic excitable media. Commun. Pure Appl. Math. 55(8), 949-1032 (2002)

[10] Blanchet, A., Dolbeault, J., Kowalczyk, M.: Stochastic Stokes' drift, homogenized functional inequalities, and large time behaviour of brownian ratchets. SIAM J. Math. Anal. 41(1), 46-76 (2009) 
[11] Budhiraja, A., Fricks, J.: Molecular motors, brownian ratchets, and reflected diffusions. DCDS(B) 6(4), 711-734 (2006)

[12] Capdeboscq, Y.: Homogenization of a neutronic critical diffusion problem with drift. Proc. R. Soc. Edinb. 132A, 567-594 (2002)

[13] Chipot, M., Hastings, S., Kinderlehrer, D.: Transport in a molecular motor system. M2AN. Math. Model. Numer. Anal. 38(6), 1011-1034 (2004)

[14] Chipot, M., Kinderlehrer, D., Kowalczyk, M.: A variational principle for molecular motors, dedicated to Piero Villaggio on the occasion of his 70th birthday. Meccanica 38(5), 505-518 (2003)

[15] Cioranescu, D., Piatnitski, A.: Homogenization of a porous medium with randomly pulsating microstructure. Multiscale Model. Simul. 5(1), 170-183 (2006) (electronic)

[16] Cioranescu, D., Piatnitski, A.: Homogenization in perforated domains with rapidly pulsing perforations. ESAIM Control Optim. Calc. Var. 9, 461-483 (2003) (electronic)

[17] Collet, P., Martinez, S.: Asymptotic velocity of one dimensional diffusions with periodic drift. J. Math. Biol. 56, 765-792 (2008)

[18] Crandall, M.G., Ishii, H., Lions, P.-L.: User's guide to viscosity solutions of second order partial differential equations. Bull. Am. Math. Soc. 27, 1-67 (1992)

[19] Doering, C., Ermentrout, B., Oster, G.: Rotary DNA motors. Biophys. J. 69(6), 2256-2267 (1995)

[20] Dolbeault, J., Kinderlehrer, D., Kowalczyk, M.: Remarks about the flashing ratchet. In: Partial differential equations and inverse problems. Contemp. Math., vol. 362, pp. 167-175. American Mathmatical Society, Providence, RI (2004)

[21] Donato, P., Piatniski, A.: Averaging of nonstationary parabolic operators with large lower order terms. In: Multiscale problems and asymptotic analysis. Gakuto Internat. Ser. Math. Sci. Appl., vol. 24, pp. 153-165. Gakkotosho, Tokyo (2006)

[22] Evans, L.C.: The perturbed test function method for viscosity solutions of nonlinear PDE. Proc. R. Soc. Edinb. 111A, 359-375 (1989)

[23] Evans, L.C., Souganidis, P.E.: A PDE approach to geometric optics for certain reaction-diffusion equations. Indiana Univ. Math J. 38, 141-172 (1989)

[24] Garnier, J.: Homogenization in a periodic and time-dependent potential. (English summary). SIAM J. Appl. Math. 57(1), 95-111 (1997)

[25] Ishii, H.: Perron's method for Hamilton-Jacobi equations. Duke Math. J. 55(2), 369-384 (1987)

[26] Jülicher, F., Ajdari, A., Prost, J.: Modeling molecular motors. Rev. Mod. Phys. 69(4), 1269-1281 (1997) 
[27] Hastings, S., Kinderlehrer, D., McLeod, J.B.: Diffusion mediated transport with a look at motor proteins. In: Recent advances in nonlinear analysis, pp. 95-111. World Scientific Publishing, Hackensack (2008)

[28] Howard, J.: Mechanics of motor proteins and the cytoskeleton. Sinauer Associates, Inc., Sunderland (2001)

[29] Kinderlehrer, D., Kowalczyk, M.: Diffusion-mediated transport and the flashing ratchet. Arch. Ration. Mech. Anal. 161(2), 149-179 (2002)

[30] Majda, A.J., Souganidis, P.E.: Large scale front dynamics for turbulent diffusion equations with separated velocity scales. Nonlinearity 7, 1-30 (1994)

[31] Moser, J.: A Harnack inequality for parabolic differential equations. Commun. Pure Appl. Math. 17, 101-134 (1964)

[32] Perthame, B.: Perturbed dynamical systems with an attracting singularity and weak viscosity limits in Hamilton-Jacobi equations. Trans. Am. Math. Soc. 317(2), 723-748 (1990)

[33] Perthame, B., Souganidis, P.E.: Asymmetric potentials and motor effect: a large deviation approach. Arch. Ration. Mech. Anal. 193(1), 153-169 (2009)

[34] Perthame, B., Souganidis, P.E.: Asymmetric potentials and motor effect: a homogenization approach. Ann. I. H. Poincaré-AN 26, 2055-2071 (2009)

[35] Peskin, C.S., Ermentrout, B., Oster, G.: The correlation ratchet: a novel mechanism for generating directed motion by ATP hydrolysis. In: Mow, V.C. et al. (eds.) Cell Mechanics and Cellular Engineering. Springer, New York (1995)

B. Perthame

INRIA (Bang) and Institute Universitaire de France,

CNRS, UMR 7598 LJLL, BC187,

Université Pierre et Marie Curie-Paris 6,

4, place Jussieu, 75252 Paris Cedex 05, France

e-mail: benoit.perthame@upmc.fr

P. E. Souganidis

Department of Mathematics,

The University of Chicago,

5734 S. University Avenue,

Chicago, Illinois 60637,

USA

e-mail: souganidis@math.uchicago.edu

Received: 16 January 2010.

Revised: 26 May 2010.

Accepted: 08 June 2010. 